\title{
Blood Product Bacterial Testing 24 Hours Prior to Transfusion
}

National Cancer Institute

\section{Source}

National Cancer Institute. Blood Product Bacterial Testing 24 Hours Prior to

Transfusion. NCI Thesaurus. Code C133327.

A qualitative immunoassay for the detection of aerobic and anaerobic gram-positive and gram-negative bacteria in leukocyte reduced apheresis platelets or pre-storage pools of up to six (6) leukocyte reduced whole blood derived platelets within 24 hours prior to transfusion. 